\title{
Christmas Tree Case Study: Computed Tomography as a Tool for Mastering Complex Real World Objects with Applications in Computer Graphics
}

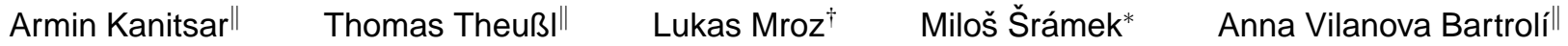 \\ Balázs Csébfalvill Jiří Hladůvkal Dominik Fleischmann ${ }^{\S}$ Michael Knapp\| Rainer Wegenkittl ${ }^{\dagger}$ \\ Petr Felkel ${ }^{\ddagger}$ Stefan Röttger ${ }^{\mathbb{I l}}$ Stefan Guthe** Werner Purgathofer ${ }^{* *}$ Meister Eduard Gröller\|
*Austrian Academy of Sciences $\quad$ Tiani Medgraph $\quad \doteqdot$ VRVis Research Center $\quad \S$ Department of Radiology
Vienna, Austria $\quad$ Austria Vienna, Austria University of Vienna \\ II VIS II Institute of Computer Graphics and Algorithms $\quad * *$ WSI/GRIS \\ University of Stuttgart \\ Vienna University of Technology \\ University of Tübingen
}
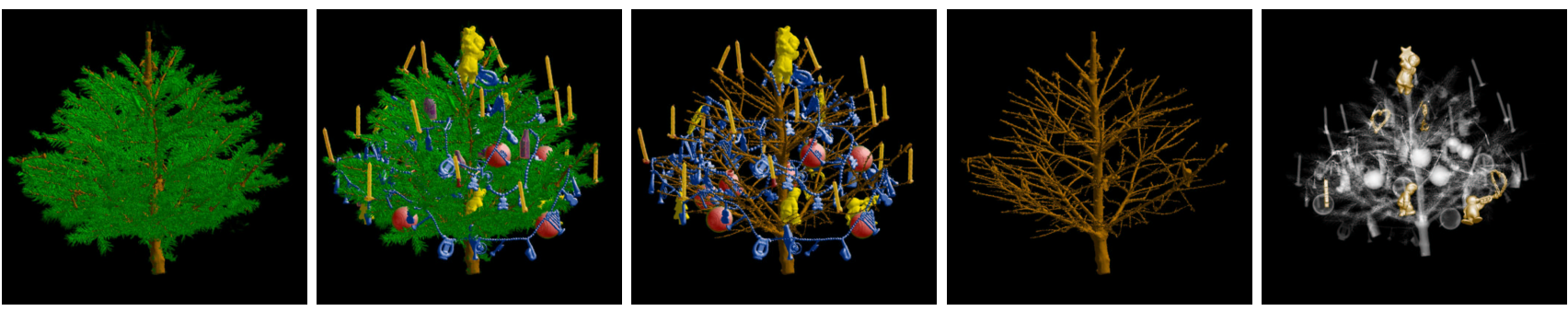

Figure 1: A short story: Before Christmas - the $25^{\text {th }}$ - left for holidays - the sad end - Christmas tree in heaven.

\section{ABSTRACT}

We report on using computed tomography (CT) as a model acquisition tool for complex objects in computer graphics. Unlike other modeling and scanning techniques the complexity of the object is irrelevant in CT, which naturally enables to model objects with, for example, concavities, holes, twists or fine surface details. Once the data is scanned, one can apply post-processing techniques for data enhancement, modification or presentation. For demonstration purposes we chose to scan a Christmas tree which exhibits high complexity which is difficult or even impossible to handle with other techniques. However, care has to be taken to achieve good scanning results with $\mathrm{CT}$. Further, we illustrate post-processing by means of data segmentation and photorealistic as well as non-photorealistic surface and volume rendering techniques.

CR Categories: I.3.3 [Computing Methodologies]: Computer Graphics-Picture/Image Generation I.6.5 [Computing Methodologies]: Simulation and Modeling-Model Development

Keywords: modeling, computed tomography, volume visualization

\footnotetext{
*Milos.Sramek@oeaw.ac.at

${ }^{\dagger}$ \{mroz,wegenkittl\}@tiani.com

†felkel@vrvis.at

§ominik.fleischmann@univie.ac.at

IIStefan.Roettger@informatik.uni-stuttgart.de

\| $\{$ kanitsar,theussl,anna,balazs,jiri,knapp,wp,meister\}@cg.tuwien.ac.at

** guthe@gris.uni-tuebingen.de
}

\section{INTRODUCTION}

In computer art, one is often faced with the task to digitize real world objects for further processing with various computer graphics tools. The commonly used methods like range scanning suffer from certain limitations since only information of the visible surfaces of objects is provided. Non-convex surfaces, an inherent property of complex objects, often cause difficulties or even cannot be handled at all. Similar arguments hold for taking a set of photographs from different viewpoints to be used for image based rendering.

With procedural modeling of existing physical objects it is difficult to come up with an accurate description of the real world object. Furthermore it requires special effort to get a physically correct model and the more complex the model is the more difficult the modeling task becomes.

Generating data sets (i.e., modeling the objects) by scanning real world objects using CT modalities is independent of model complexity and physical correctness is automatically achieved. Different structures within the data set can be segmented and the interior can be examined as CT provides volumetric descriptions. On the other hand, CT scanners are rather expensive and not easily accessible. In this paper we describe our experiences of using CT to model a decorated Christmas tree, a very complex object with many different structures and small details.

\section{Related Work}

Computed tomography has been used for non-destructive investigations of trees [4]. In order to do this, mobile CTs have been built which extract single slices. This way diseases of trees are detected with adequate precision. 
State-of-the-art interactive modeling tools today are either polygon or patch oriented (Maya, Truespace). However, manipulation of the underlying data structures (polygonal meshes, NURBS) is often not versatile enough to fulfill the artist's demands. Therefore, in the early stages of the design often clay models are created, which are subsequently digitized by means of range scanners [8].

There is of course a lot of literature on laser range scanning objects. Particularly interesting for the visualization community is the work by Levoy et al. [6], who describe their efforts to digitize large objects at high resolution with mobile laser range scanning hardware. However, for objects with a high degree of complexity this approach is not applicable.

\section{Dataset Acquisition}

To obtain a realistic model of a Christmas tree, we decided to use $\mathrm{CT}$. The following section describes the generation process and preprocessing of the data set.

\section{Model Preparation:}

Firstly, special care had to be taken in the preparation of the real model to assume a high data set quality. Especially, no metal was allowed to be used, as this would result in artifacts. Therefore the sweets were unwrapped and metal hooks replaced (see Figure 2). Due to the limited diameter of the CT gantry, the size of the tree must not exceed $50 \mathrm{~cm}$. Furthermore the proportions of the Christmas tree decoration and the small conifer should match those of a real Christmas tree. Therefore special items, as small candles for birthday cakes, were used.

\section{Scanning Process:}

The CT acquisition protocoll had to be specifically designed as neither presets nor protocols are available for small trees. The Christmas-tree model was scanned with a Siemens Somatom Plus 4 Volume Zoom Multislice-CT scanner at the general hospital in Vienna. A tube voltage of $140 \mathrm{kV}$ and a tube current of $100 \mathrm{mAs}$ was used during the 40 s scan. The collimation was $4 \times 1 \mathrm{~mm}$, the table increment was $3 \mathrm{~mm} / 360^{\circ}$ gantry rotation. Transverse sections with a nominal slice width of $1.25 \mathrm{~mm}$ were reconstructed at $0.5 \mathrm{~mm}$ intervals using a medium/sharp (B40) reconstruction kernel. A dataset of $512 \times 512 \times 998$ voxels was reconstructed from a field of view of $476 \mathrm{~mm} \times 476 \mathrm{~mm} \times 499 \mathrm{~mm}$.

\section{Preprocessing:}

Due to low noise and good spatial resolution of the data set no further preprocessing and enhancement was necessary. Limitations of some rendering tools required seperate 8 bit voxel-valued data sets.

The resulting data were originally stored in DICOM format, which is the standard format in medical imaging. For scientific and experimental purposes more handy file formats are desirable. Therefore, the data set was converted (and is publicly available therein) to the dat and $\mathrm{f} 3 \mathrm{~d}$ formats $[14,11]$. The first six bytes of the dat format represent the data set dimension. Each following pair of bytes represent one voxel. The $\mathrm{f} 3 \mathrm{~d}$ format is more general and stores all the necessary data attributes in an easy to read header, followed by a preview image and the compressed 3D data itself.

Segmentation of tomographic data sets is traditionally depending on the goals, either very simple or very complicated. The simple task in our case was classification of the foreground voxels (the tree itself with decorations) with respect to the background. This task was accomplished easily by thresholding. The hard segmentation task was to separate various tissues of the tree (trunk and needles) and decorations. For this purpose we used two segmentation tools, ISEG [9] and the segmentation functionality of JVision (a medical 3D workstation from TIANI MedGraph [13]). ISEG is a general purpose interactive system for segmentation of $3 \mathrm{D}$ data sets based on the assumption of spatial object homogeneity. Such

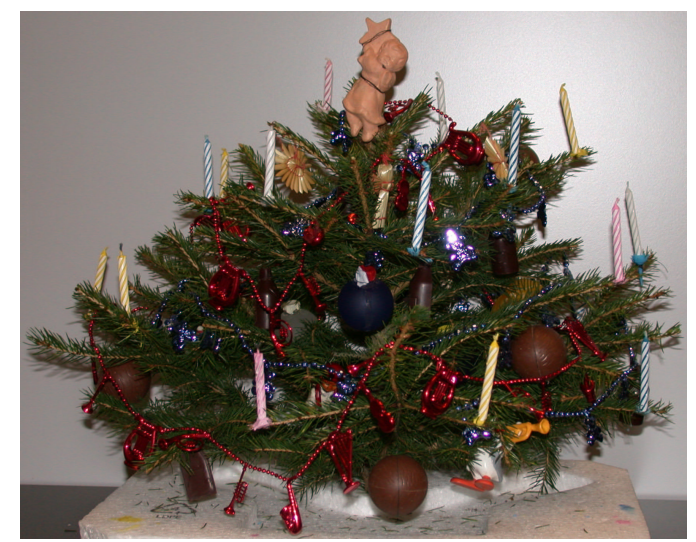

Figure 2: A picture of the Christmas tree.

objects can be identified by thresholding. Several objects can be selected even if their density ranges overlap. Therefore, tools are provided (connected component labeling, morphological—erosion, dilation and smoothing — and logical—voxel-wise AND, OR, XORoperations) to separate such objects. 15 different objects were labeled, some of them representing several instances of similar but spatially disconnected decorations (e.g., candles or balls). A similar functionality was available for segmentation using JVision. Separating objects with similar value ranges and close spatial locations is often difficult to handle with just threshold based and morphological methods. Therefore, a local water-shed method [2] was applied to split and divide masks containing multiple objects.

\section{Visualization ALgORItHMS}

In the following the results of different volume visualization methods are presented.

\section{Shaded Surface Display:}

Figure 3 (left) shows polygon rendering using OpenGL hardware acceleration. The iso-surfaces at a value of $-944 \mathrm{HU}$ (Hounsfield Units) were extracted from the data set using an extended marching cubes algorithm. The internal structure of the decoration is represented with opaque surfaces.

Object labels and masks, which were the result of the segmentation step were further used for high quality rendering by means of ray tracing. Smooth object surfaces were obtained directly from the label volume by a first-order interpolation and thresholding [10], while the normals, necessary for realistic surface shading, were estimated directly from the original CT data by central differences and trilinear interpolation (Figure 3, middle). The Chessboard distance ray traversal technique [12] was used to speed up the rendering.

\section{Direct Volume Rendering:}

In Figure 3 (right) software raycasting is presented using a sharp transfer function showing an iso-surface at $-954 \mathrm{HU}$.

Interactive direct volume rendering running at 5 to 10 frames per second, on a Pentium 4 based system using a GeForce4 Ti4600 graphics card is used in Figure 4 to the left. This approach is based on real-time decompression of a wavelet encoded multiresolution hierarchy and multiresolution volume rendering [3]. The images are rendered using lossless compression at a ratio of about 3.9:1.

Figure 6 demonstrates volume rendering using a view-dependent hierarchical octree representation and a screen space error of less than one pixel. Pre-integration was used to render the data with exactly projected cells. 
Figure 1 shows images of the Christmas tree rendered using the RTVR library [7], at about 3-4 frames per second on a PIII/1.2GHz machine (full spatial resolution of the dataset, $512 \times 512$ image). RTVR allows to combine different compositing modes for different objects like surface rendering for the angels and summation (X-ray) for the remaining objects in image 5 of Figure 1. As RTVR is a pure Java solution the tree can be viewed interactively using an RTVR applet at this paper's web-page [14].

Figure 4 (middle) shows non-photorealistic direct volume rendering using the "Bubble Model" [1]. Iso-surfaces are rendered as thin semi-transparent membranes similarly to blown soap bubbles. The approach is a fast previewing technique for volumetric data which does not require a time consuming transfer function specification to visualize internal structures. Originally developed for medical data the method also works for the scanned data set in quickly illustrating the most important features.

\section{Arboroscopy:}

Virtual endoscopy is used to inspect tubular objects of volumetric data sets. Applied to our data set, the term arboroscopy most illustratively describes this technique. A seven-fold branching is shown in Figure 4 (right). As branches of such high degree rarely exist in common endoscopic applications, this dataset provides an interesting worst-case test scenario.

\section{Curved Planar Reformation (CPR):}

Curved Planar Reformation is a visualization technique used to inspect vascular structures [5]. The re-sampled plane in Figure 5 is generated from the central axis of a branch of the tree. Thus the stability and correctness of the CPR algorithm could be investigated exemplarily on this special data set.

\section{Conclusions}

We have presented a straightforward technique for modeling of complex shapes by means of tomographic scanning. The limits of its applicability are given by the scanner properties, which define the outer and inner scale of the representable object: one can scan objects with dimensions up to about $500 \mathrm{~mm}$ with small details at about $1 \mathrm{~mm}$ or even less. Some materials (i.e., metal) cause artifacts and therefore should be, if possible, avoided. Color information is lost during the scan process. Up to now CT modalities are expensive and restricted in accessibility.

On the other hand this model acquisition method provides important properties. The data acquisition is independent from the object complexity and both surfaces and object interiors are represented. Different types of material are distinguishable according to the material dependent attenuation. Computed Tomography produces high resolution data sets. Even small features, as the needles of the presented data set, are still properly sampled.

Finally, a contribution of this work is to provide the visualization community with a high resolution data set with some interesting properties. It includes rather large and smooth objects like the figure on top of the tree as well as a lot of fine details like the needles. It is usable as a test data set for several applications which we showed by applying various visualization algorithms. The Christmas tree data set is publicly available from our web site [14].

\section{ACKNOWLEDGEMENTS}

The work presented in this publication has been partly funded by: AngioVis - Visualization Tools for Peripheral CT-Angiography project (FWF-P15217-INF), supported by the Fonds zur Förderung der wissenschaftlichen Forschung, Austria; ADAPT project (FFF-804544), supported by Tiani Medgraph and the Forschungsförderungsfonds für die gewerbliche Wirtschaft, Austria; the VRVis research center, Vienna supported by the K plus Programm. Special thanks to Martin Kampel and Robert Sablatnig from the Pattern Recognition and Image Processing Group, Vienna University of Technology, for laser range scanning the Christmas tree (and telling us in advance it would not work). Thanks to Theresia Fasser for involuntarily donating the tree that started it all.

\section{REFERENCES}

[1] B. Csébfalvi and E. Gröller. Interactive volume rendering based on a "bubble model". In GI 2001, pages 209-216, 2001.

[2] P. Felkel, M. Bruckwschwaiger, and R. Wegenkittl. Implementation and complexity of the watershed-from-markers algorithm computed as a minimal cost forest. In Eurographics 2001, volume 20 of Computer Graphics Forum, pages 26-35, Manchester, United Kingdom, 2001.

[3] S. Guthe and W. Strasser. Real-time decompression and visualization of animated volume data. In IEEE Visualization 2001, pages 349-356. ACM, 2001.

[4] A. Habermehl and H.-W. Ridder. Applications of computerized tomography in forest and tree sciences. In International Symposium on Computerized Tomography for Industrial Applications, 1994.

[5] A. Kanitsar, R. Wegenkittl, P. Felkel, D. Fleischmann, D. Sandner, and E. Gröller. Computed Tomography Angiography: A Case Study of Peripheral Vessel Investigation. In IEEE Visualization 2001, pages 477-480. ACM, 2001.

[6] M. Levoy, K. Pulli, B. Curless, S. Rusinkiewicz, D. Koller, L. Pereira, M. Ginzton, S. Anderson, J. Davis, J. Ginsberg, J. Shade, and D. Fulk. The digital Michelangelo project: 3D scanning of large statues. In Siggraph 2000, Computer Graphics Proceedings, pages 131-144. ACM, 2000.

[7] L. Mroz and H. Hauser. RTVR - a flexible Java library for interactive volume rendering. In IEEE Visualization 2001, pages 279-286. ACM, 2001.

[8] R. N. Perry and S. F. Frisken. Kizamu: A system for sculpting digital images. In Eugene Fiume, editor, Siggraph 2001, Computer Graphics Proceedings, Annual Conference Series, pages 47-56. ACM, 2001.

[9] M. Šrámek. Interactive segmentation of tissues for medical imaging. In Václav Hlaváč and Tomáš Pajdla, editors, Czech Pattern Recognition Workshop '93, pages 164-171, Temešvár u Písku, Czech Republic, 1993.

[10] M. Šrámek. Fast surface rendering from raster data by voxel traversal using chessboard distance. In IEEE Visualization'94, pages 188-195. ACM, 1994.

[11] M. Šrámek and L. I. Dimitrov. $\mathrm{f} 3 \mathrm{~d}$ - a file format and tools for storage and manipulation of volumetric data sets. In $1^{s t}$ International Symposium on 3D Data Processing, Visualization and Transmission, pages 368-371, Padova, Italy, 2002. IEEE CS.

[12] M. Šrámek and A. Kaufman. Fast ray-tracing of rectilinear volume data using distance transforms. Transactions on Visualization and Computer Graphics, 6(3):236-252, 2000.

[13] Tiani MedGraph. JVision/Spacevision diagnostic workstation, 2002. http://www.tiani.com/.

[14] XMT. Christmas Tree Web Page, 2001. http: //ringlotte.cg.tuwien.ac.at/datasets/ XMasTree/XMasTree.html. 

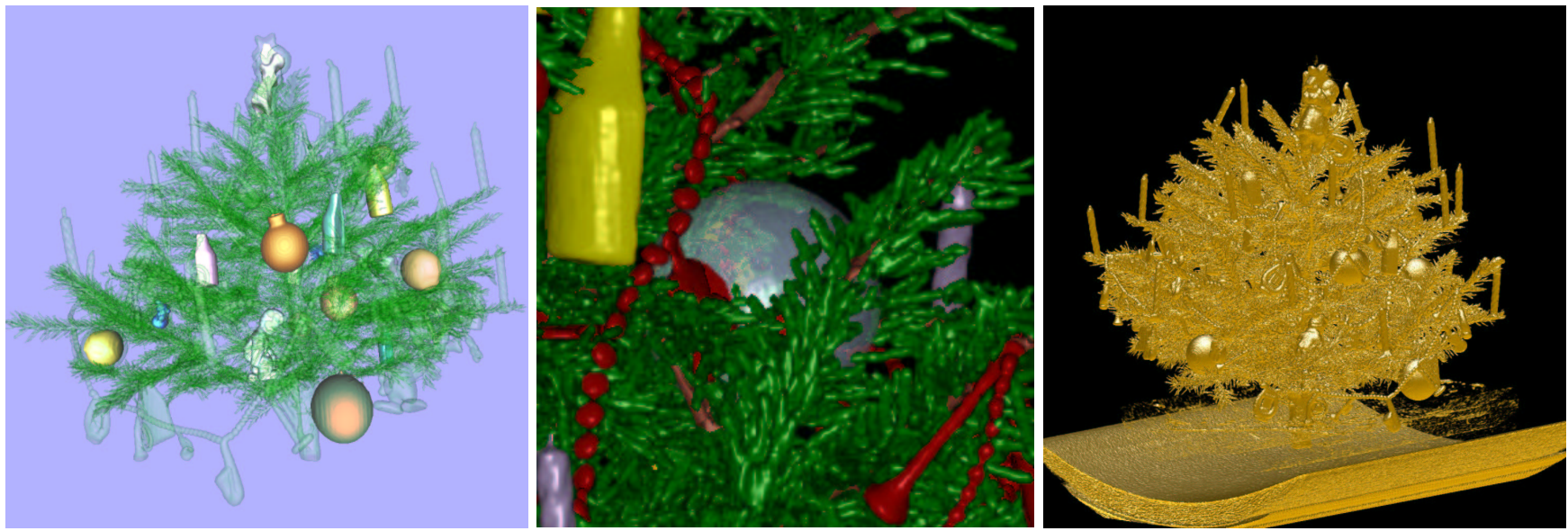

Figure 3: From left to right: OpenGL polygon rendering based on marching cubes surface extraction. Ray tracing of the segmented tree. Direct volume rendering: ray casting.
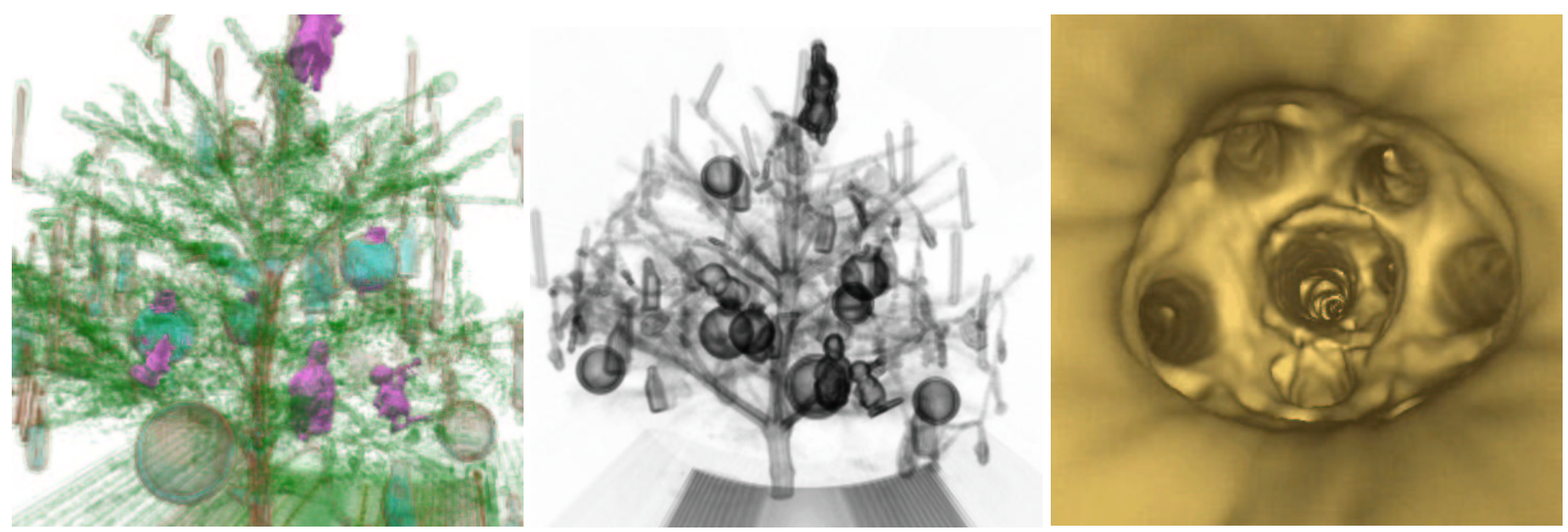

Figure 4: From left to right: Interactive wavelet encoded multiresolution volume rendering. Non-photorealistic rendering using the "Bubble Model". View of a seven-fold branch in a virtual arboroscopy.

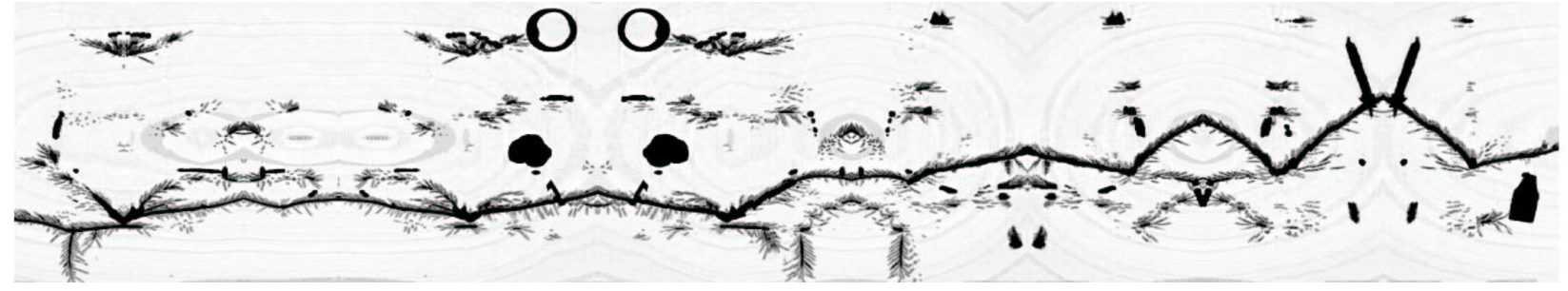

Figure 5: A Curved Planar Reformation of the XMas-Tree dataset.
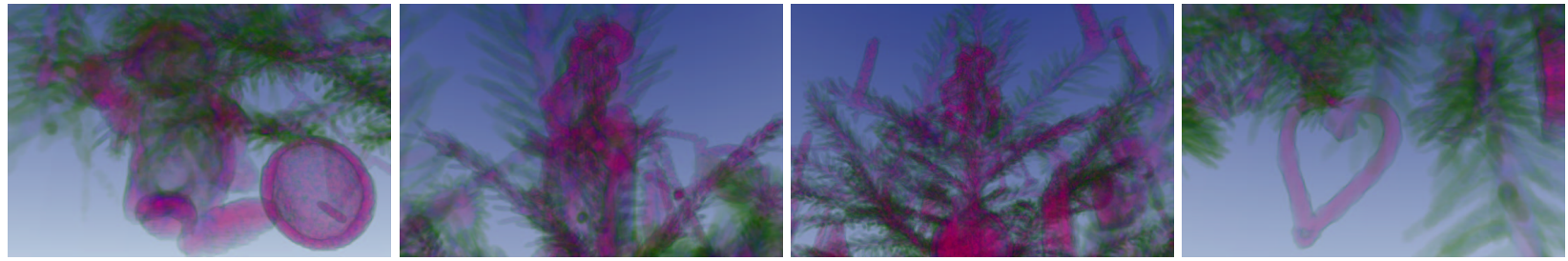

Figure 6: Volume rendering using a view-dependent hierarchical octree representation. 